Article

\title{
Self-Similar Inverse Semigroups from Wieler Solenoids
}

\author{
Inhyeop Yi \\ Department of Mathematics Education, Ewha Womans University, Seoul 03760, Korea; yih@ewha.ac.kr
}

Received: 16 January 2020; Accepted: 13 February 2020; Published: 17 February 2020

\begin{abstract}
Wieler showed that every irreducible Smale space with totally disconnected local stable sets is an inverse limit system, called a Wieler solenoid. We study self-similar inverse semigroups defined by s-resolving factor maps of Wieler solenoids. We show that the groupoids of germs and the tight groupoids of these inverse semigroups are equivalent to the unstable groupoids of Wieler solenoids. We also show that the $C^{*}$-algebras of the groupoids of germs have a unique tracial state.
\end{abstract}

Keywords: Smale space; Wieler solenoid; self-similar inverse semigroup; limit solenoid; groupoid of germs; tight groupoid; unstable $C^{*}$-algebra

MSC: 46L05; 46L55; 37D20

\section{Introduction}

The purpose of this work is to study groupoids of germs and tight groupoids on a certain class of Smale spaces. Wieler [1] showed that irreducible Smale spaces with totally disconnected local stable sets can be realized as stationary inverse limit systems satisfying certain conditions, now called Wieler solenoids [1-4].

Wieler solenoids have some interesting properties related to inverse semigroups. Existence of totally disconnected local stable sets induce a canonical SFT (subshift of finite type) cover of a Wieler solenoid whose factor map is an s-resolving map. Nekrashevych defined self-similar inverse semigroups, called adjacency semigroups, on Smale spaces with s-resolving factor maps [5-7]. The limit solenoid defined by the adjacency semigroup of a Smale space is topologically conjugate to the original Smale space [7]. In the present paper, we use adjacency semigroups to study unstable equivalence relations on Wieler solenoids.

Our study is very much inspired by a work of Exel, Gonçalves and Starling [8] in the special case of substitution tilings. In [8], they showed that, for a tiling system with certain conditions, the groupoid of germs of a tiling inverse semigroup, the tight groupoid of the tiling inverse semigroup and the unstable groupoid of the tiling system are all isomorphic to each other. We follow [8] and introduce a Wieler solenoid with its adjacency semigroup to obtain the equivalence of the groupoid of germs of the adjacency semigroup, the tight groupoid of the adjacency semigroup and the unstable groupoid of the Wieler solenoid.

This paper is organized in the following manner. After providing background materials for Smale spaces, Wieler solenoids and inverse semigroups, in Section 4 we introduce the adjacency semigroup. Then we establish equivalence among the groupoids of Wieler solenoids and their adjacency semigroups in Section 5 . In Section 6, we show that $C^{*}$-algebra of the groupoid of germs has a unique tracial state. We close with a section providing an example. 


\section{Smale Spaces and Wieler Solenoids}

\subsection{Smale Spaces}

We review the definition and some well-known properties about Smale spaces and their associated $C^{*}$-algebras. We refer to [9-12] for more details.

Definition 1 ([10]). Let $(X, d)$ be a compact metric space and $f: X \rightarrow X$ a homeomorphism. Assume that we have constants $0<\lambda<1, \epsilon_{0}>0$ and a continuous map, called the bracket map,

$$
[\cdot, \cdot]:\left\{(x, y) \in X \times X \mid d(x, y) \leq \epsilon_{0}\right\} \rightarrow X, \quad(x, y) \mapsto[x, y] .
$$

Then $\left(X, d, f,[\cdot, \cdot], \lambda, \epsilon_{0}\right)$ is called a Smale space if the following conditions hold:

$$
\begin{aligned}
& {[x, x]=x,} \\
& {[[x, y], z]=[x, z] \text { when both sides are defined, }} \\
& {[x,[y, z]]=[x, z] \text { when both sides are defined, }} \\
& {[f(x), f(y)]=f([x, y]) \text { when both sides are defined, }} \\
& d(f(x), f(y)) \leq \lambda d(x, y) \text { when }[x, y]=y, \text { and } \\
& d\left(f^{-1}(x), f^{-1}(y)\right) \leq \lambda d(x, y) \text { when }[x, y]=x .
\end{aligned}
$$

We denote a Smale space by $(X, f)$, the other data are taken implicitly.

For $x \in X$ and $0<\epsilon \leq \epsilon_{0}$, we define

$$
\begin{aligned}
& X^{s}(x, \epsilon)=\{y \in X \mid d(x, y)<\epsilon \text { and }[x, y]=y\} \text { and } \\
& X^{u}(x, \epsilon)=\{y \in X \mid d(x, y)<\epsilon \text { and }[y, x]=y\} .
\end{aligned}
$$

We call $X^{s}(x, \epsilon)$ and $X^{u}(x, \epsilon)$ a local stable set of $x$ and a local unstable set of $x$, respectively.

For a Smale space $(X, f)$, define

$$
G_{u, 0}=\left\{(x, y) \in X \times X \mid y \in X^{u}\left(x, \epsilon_{0}\right)\right\}
$$

and let

$$
G_{u}=\bigcup_{n=0}^{\infty}(f \times f)^{n}\left(G_{u, 0}\right) .
$$

Then $G_{u}$ is an equivalence relation on $X$, called the unstable equivalence and denoted by $\sim_{u}$. It is easy to observe that

$$
x \sim_{u} y \text { if and only if } d\left(f^{-n}(x), f^{-n}(y)\right) \rightarrow 0 \text { as } n \rightarrow \infty .
$$

Each $(f \times f)^{n}\left(G_{u, 0}\right)$ is given the relative topology of $X \times X$, and $G_{u}$ is given the inductive limit topology. It is not difficult to verify that $G_{u}$ is a locally compact Hausdorff principal groupoid. The Haar system for $G_{u}$ is described in ([11], 3.c). The groupoid $C^{*}$-algebra $C^{*}\left(G_{u}\right)$ is called the unstable algebra for $(X, f)$.

\subsection{Wieler Solenoids}

Wieler [1] characterized irreducible Smale spaces with totally disconnected local stable sets as stationary inverse limits of spaces.

Definition 2. Suppose that $\left(Y, d_{Y}\right)$ is a compact metric space and that $g: Y \rightarrow Y$ is a continuous surjective map. We say that $(Y, g)$ satisfies Wieler's axiom if there are constants $\beta>0, K \in \mathbb{N}^{+}$and $0<\gamma<1$ such that the following hold: 
Axiom 1 If $v, w \in Y$ satisfy $d_{Y}(v, w) \leq \beta$, then

$$
d_{Y}\left(g^{K}(v), g^{K}(w)\right) \leq \gamma^{K} d_{Y}\left(g^{2 K}(v), g^{2 K}(w)\right) .
$$

Axiom 2 For every $v \in Y$ and $0<\epsilon \leq \beta$,

$$
g^{K}\left(B\left(g^{K}(v), \epsilon\right)\right) \subseteq g^{2 K}(B(v, \gamma \epsilon))
$$

where $B(y, r)$ denotes a closed ball.

Definition 3. Suppose that $(Y, g)$ satisfies Wieler's axioms. Then we define the inverse limit

$$
X=\lim _{\longleftarrow}(Y, g)=\left\{\left(v_{0}, v_{1}, v_{2}, \ldots\right) \in Y^{\mathbb{N}}: g\left(v_{n+1}\right)=v_{n} \text { for every } n \geq 0\right\}
$$

along with the induced map $\varphi: X \rightarrow X$ given by

$$
\left(v_{0}, v_{1}, v_{2}, \ldots\right) \mapsto\left(g\left(v_{0}\right), v_{0}, v_{1}, v_{2}, \ldots\right) .
$$

We call $(X, \varphi)$ the Wieler solenoid associated to $(Y, g)$.

Theorem 1. [1] If $(Y, g)$ satisfies Wieler's axioms, then the associated Wieler solenoid is a Smale space with totally disconnected local stable sets. Moreover, if $(Y, g)$ is irreducible, so is the associated Wieler solenoid.

Conversely, if $(X, f)$ is an irreducible Smale space with totally disconnected local stable sets, then there is an irreducible dynamical system $(Y, g)$ satisfying Wieler's axioms such that $(X, f)$ is topologically conjugate to the Wieler solenoid associated to $(Y, g)$.

Recall that, for Smale spaces $\left(A, f_{a}\right)$ and $\left(B, f_{b}\right)$, a factor map is a continuous onto map $\pi: A \rightarrow B$ satisfying $f_{b} \circ \pi=\pi \circ f_{a}$. A factor map $\pi$ is s-resolving if $\left.\pi\right|_{X^{s}(x, \epsilon)}$ is one-to-one for every $x \in A$ and some $\epsilon>0$. See $[1,13]$ for more details.

Theorem 2 ([13], Corollary 1.3). If $(X, f)$ is an irreducible Wieler solenoid, then there is an irreducible subshift of finite type $(\Sigma, \sigma)$ and an s-resolving factor map $\pi:(\Sigma, \sigma) \rightarrow(X, f)$.

\section{Self-Similar Inverse Semigroups}

\subsection{Inverse Semigroups}

An inverse semigroup is a semigroup $H$ such that for every $h \in H$, there is a unique element $h^{*} \in H$, called the inverse of $h$, satisfying

$$
h h^{*} h=h \text { and } h^{*} h h^{*}=h^{*} .
$$

We assume that $H$ has a unit element 1 and a zero element 0 with the property

$$
1 h=h 1=h \text { and } 0 h=h 0=0 \text { for every } h \in H .
$$

An element $h \in H$ is called an idempotent if $h^{2}=h$. We denote the set of all idempotents in $H$ by $E$.

Example 1 ([14], [Definition 5.2). ] Let X be a topological space and define

$$
\text { pHomeo }(X)=\{h: U \rightarrow V \mid U, V \subset X \text { are open, and } h \text { is a homeomorphism }\} \text {. }
$$


Then, $\mathrm{pHomeo}(X)$ is an inverse semigroup: Its binary operation is given by composition, for $h_{1}, h_{2} \in$ $\mathrm{pHomeo}(X)$,

$$
h_{1} h_{2}=h_{1} \circ h_{2}: h_{2}^{-1}\left(D o m h_{1} \cap I m h_{2}\right) \rightarrow h_{1}\left(D o m h_{1} \cap \operatorname{Im} h_{2}\right) .
$$

The inverse is given by $h^{*}=h^{-1}$, the unit element is $I d_{X}$, the 0 element is the trivial map between empty sets, and $h$ is an idempotent if and only if $h=I d_{U}$ for some open subset $U$ of $X$.

An element $h \in \mathrm{pHomeo}(X)$ is called a partial homeomorphism of $X$.

For an inverse semigroup $H$ and a topological space $X, H$ is said to act on $X$ if there is a semigroup homomorphism $\theta: H \rightarrow$ pHomeo $(X)$ that preserves the unit element and the zero element. By abuse of notation, we denote $\theta(h)$ by $h$ itself.

\subsection{Groupoids of Germs}

Suppose that an inverse semigroup $H$ acts on a locally compact Hausdorff space $Y$. The groupoid of germs of $H$, denoted $\Gamma$, is the set of equivalence classes of pairs $(h, u)$ such that $h \in H$ and $u \in \operatorname{Dom} h$. Two pairs $(h, u)$ and $(g, v)$ are equivalent to each other if and only if $u=v$ and there is an idempotent $e \in E$ such that $u \in$ Dom $e$ and $h e=g e$. The equivalence class of $(h, u)$ is denoted by $[h, u]$ and called the germ of $h$ at $u$.

The domain and range maps of $\Gamma$ are

$$
d([h, u])=u \text { and } r([h, u])=h(u) .
$$

The set of composable pairs of $\Gamma_{H}$ is

$$
\Gamma^{(2)}=\{([h, u],[g, v]) \in \Gamma \times \Gamma: g(v)=u\},
$$

and the groupoid composition and inversion are given as

$$
[h, u] \cdot[g, v]=[h g, v] \text { and }[h, u]^{-1}=\left[h^{*}, h(u)\right] .
$$

The unit space of $\Gamma$ is

$$
\Gamma^{(0)}=\{[e, u]: e \in E, u \in \operatorname{Dom} e\},
$$

which is identified with $Y$ via the bijective map $[e, u] \mapsto u$.

A topology on $\Gamma$ is given as follows: For an $h \in H$ and any open set $U \subseteq \operatorname{Dom} h^{*} h$, let

$$
O(h, U)=\{[h, u]: u \in U\} .
$$

Then the collection of all $O(h, U)$ forms a basis for a topology on $\Gamma$, which makes $\Gamma$ a locally compact étale groupoid [15].

\subsection{Tight Groupoids of Inverse Semigroups}

Every material in this subsection is taken from [16]. Suppose that $H$ is an inverse semigroup and that

$$
E=\left\{e \in H: e=e^{*}=e^{2}\right\}
$$

is the set of idempotents of $H$. Then the natural partial order on $H$ (see [17], Lemma 1.4.6) is applied on $E$;

$$
\text { for every } e_{1}, e_{2} \in E, e_{1} \leq e_{2} \Longleftrightarrow e_{1}=e_{2} e_{1} \text {. }
$$

A filter in $E$ is a nonempty subset $C \subset E$ such that

1. $0 \notin C$,

2. $e_{1} \in C$ and $e_{1} \leq e_{2}$ imply $e_{2} \in C$, and 
3. for any elements $e_{1}, e_{2} \in C$, there is an element $e_{3} \in C$ such that $e_{3} \leq e_{1}, e_{2}$.

An ultrafilter is a filter that is not properly contained in any other filter.

A character is a non-zero map $\phi: E \rightarrow\{0,1\}$ such that

1. $\phi(0)=0$ and

2. $\phi\left(e_{1} e_{2}\right)=\phi\left(e_{1}\right) \phi\left(e_{2}\right)$.

The set of characters on $E$ is denoted $\hat{E}_{0}$, which is a locally compact Hausdorff space with the pointwise convergence topology.

There is a one-to-one correspondence between $\hat{E}_{0}$ and the set of filters in $E$ :

Lemma 1 ([16], p. 251). If $\phi$ is a character, then the set

$$
C_{\phi}=\{e \in E: \phi(e)=1\}
$$

is a filter. On the other hand, if $C$ is a filter, then $\phi_{C}: E \rightarrow\{0,1\}$ defined by

$$
\phi_{C}(e)= \begin{cases}1 & \text { if } e \in C \\ 0 & \text { otherwise }\end{cases}
$$

is a character.

The set of characters on $E$ coming from ultrafilters is denoted by $\hat{E}_{\infty}$, and its closure in $\hat{E}_{0}$ is denoted by $\hat{E}_{\text {tight }}$. A subbasis for the subspace topology on $\hat{E}_{\infty}$ consists of sets of the form

$$
D_{e}=\left\{\phi \in \hat{E}_{\infty}: \phi(e)=1\right\} \text { and } \Delta_{e}=\left\{\phi \in \hat{E}_{\infty}: \phi(e)=0\right\}
$$

where $e \in E([16], \S 10)$.

For an inverse semigroup $H$ and its corresponding $\hat{E}_{t i g h t}$, there is a natural action, denoted $\theta$, of $H$ on $\hat{E}_{\text {tight }}$ : For each idempotent $e \in E$, let

$$
D_{e}=\left\{\phi \in \hat{E}_{\text {tight }}: \phi(e)=1\right\} .
$$

For every $h \in H$, the action $\theta_{h}: D_{h^{*} h} \rightarrow D_{h h^{*}}$ is defined by

$$
\phi \mapsto \theta_{h}(\phi) \text { such that } \theta_{h}(\phi)(e)=\phi\left(h^{*} e h\right) \text { for every } e \in E .
$$

The groupoid of germs of this action is called the tight groupoid of $H$ and is denoted $\mathcal{G}_{\text {tight }}$. The groupoid $C^{*}$-algebra of $\mathcal{G}_{\text {tight }}$ is called the tight $C^{*}$-algebra of $H$.

\subsection{Self-Similar Inverse Semigroups and Limit Solenoids}

First, we remark on an odd fact regarding one-sided and two-sided subshift of finite type (SFT): While a one-sided SFT $\left(\Sigma^{+}, \sigma^{+}\right)$has the the shift map $\sigma^{+}$given by

$$
\sigma^{+}: a_{0} a_{1} a_{2} \cdots \mapsto a_{1} a_{2} \cdots,
$$

the shift map on its corresponding two-sided SFT $(\Sigma, \sigma)$ is

$$
\sigma: \cdots a_{-2} a_{-1} \cdot a_{0} a_{1} \cdots \mapsto \cdots a_{-3} a_{-2} \cdot a_{-1} a_{0} \cdots .
$$

Note that $\sigma^{+}$and $\sigma$ act on different directions. 
It is a well-known fact that $\left(\Sigma^{+}, \sigma^{+}\right)$and $(\Sigma, \sigma)$ are compact metric spaces whose basis is given as follows: Let $Y$ be either $\Sigma^{+}$or $\Sigma$, and $\mathcal{B}_{n}(Y)$ the set of allowed $n$-blocks in $Y$. For every $\alpha \in \mathcal{B}_{n}(Y)$, define the cylinder set $C_{k}(\alpha)$ as

$$
C_{k}(\alpha)=\left\{w=\cdots a_{i} a_{i+1} \cdots \in Y: a_{k} \cdots a_{k+n-1}=\alpha\right\}
$$

Then each cylinder set is a clopen subset of $Y$, the collection of cylinder sets is a countable basis of $Y$, and $\left\{C_{0}(\alpha)\right\}$ is a subbasis of $Y$. We refer to [18] for more details on SFTs.

Definition 4 ([5], Definition 3.6). Suppose that $\left(\Sigma^{+}, \sigma^{+}\right)$is a one-sided SFT on a finite alphabet $\mathcal{A}$ and that $H$ is an inverse semigroup acting on $\Sigma^{+}$. We say that $H$ is a self-similar inverse semigroup if, for each $h \in H$, Dom $h$ is a clopen subset of $\Sigma^{+}$, and there exist a number $n \in \mathbb{N},\left\{a_{1}, \ldots, a_{n}\right\} \subset \mathcal{A},\left\{b_{1}, \ldots, b_{n}\right\} \subset \mathcal{A}$ and $\left\{g_{1}, \ldots, g_{n}\right\} \subset H$ such that the sets Dom $g_{i}$ are disjoint, Dom $h=\bigcup_{i=1}^{n} a_{i} \operatorname{Dom} g_{i}$, and for every $w \in \operatorname{Dom} g_{i}$ we have $h\left(a_{i} w\right)=b_{i} g_{i}(w)$.

We need the next property to make the limit solenoids Smale spaces. See [5,6] for self-similar inverse semigroups and $[7,19,20]$ for self-similar groups.

Definition 5 ([5], Definition 9.8). A self-similar inverse semigroup $H$ acting on $\Sigma^{+}$is said to be contracting if there is a finite set $\mathcal{N} \subset H$ such that for each $h \in H$ there exist numbers $k, l \in \mathbb{N},\left\{\alpha_{1}, \ldots, \alpha_{k}\right\} \subset$ $\mathcal{B}_{l}\left(\Sigma^{+}\right),\left\{\beta_{1}, \ldots, \beta_{k}\right\} \subset \mathcal{B}_{l}\left(\Sigma^{+}\right)$and $\left\{g_{1}, \ldots, g_{k}\right\} \subset \mathcal{N}$ such that the sets Dom $g_{i}$ are disjoint, Dom $h=$ $\bigcup_{i=1}^{k} \alpha_{i} \operatorname{Dom} g_{i}$, and for every $w \in \operatorname{Dom} g_{i}$ we have $h\left(\alpha_{i} w\right)=\beta_{i} g_{i}(w)$.

${ }^{i=1}$ We call the smallest set $\mathcal{N}$ satisfying the above conditions the nucleus of $H$.

Suppose that $\left(\Sigma^{+}, \sigma^{+}\right)$is a one-sided subshift of finite type, that $(\Sigma, \sigma)$ is its corresponding two-sided SFT, and that $H$ is a contracting self-similar inverse semigroup acting on $\Sigma^{+}$. Two elements $\cdots a_{-2} a_{-1} \cdot a_{0} a_{1} \cdots$ and $\cdots b_{-2} b_{-1} \cdot b_{0} b_{1} \cdots$ in $\Sigma$ are asymptotically equivalent if for every $n \in \mathbb{Z}$ there is an $h_{n} \in \mathcal{N}$ such that

$$
h_{n}\left(a_{n} a_{n+1} \cdots\right)=b_{n} b_{n+1} \cdots .
$$

Then the asymptotic equivalence is an equivalence relation on $\Sigma$, and the quotient of $\Sigma$ by this equivalence relation is a finite-dimensional metrizable space ([7], Proposition 3.5). We denote the quotient space of $\Sigma$ by the asymptotic equivalence relation by $S_{H}$. As the shift map $\sigma: \Sigma \rightarrow \Sigma$ agrees with the asymptotic equivalence relation, the shift map $\sigma$ induces a natural homeomorphism on $S_{H}$, which is also denoted by $\sigma$.

Definition $6([5,7])$. Let $\left(\Sigma^{+}, \sigma^{+}\right),(\Sigma, \sigma)$ and $H$ be as above. The dynamical system $\left(S_{H}, \sigma\right)$ is called the limit solenoid of $H$.

\section{Adjacency Semigroups of Wieler Solenoids}

We follow ([7], §4) and ([1], §4) to construct a contracting self-similar inverse semigroup $H$ acting on an SFT of an irreducible Wieler solenoid $(X, f)$. This semigroup is called the adjacency semigroup of $(X, f)$.

First, we recall that a nonempty set $R \subseteq X$ is called a rectangle if $R=\overline{\operatorname{Int}(R)}$ and $[x, y] \in R$ whenever $x, y \in R$. For a rectangle $R$ and $x \in R$, we denote $X^{S}(x, R)=X^{S}\left(x, \epsilon_{0}\right) \cap R$ and $X^{u}(x, R)=$ $X^{u}\left(x, \epsilon_{0}\right) \cap R$. See [1,7] for details.

Lemma 2 ([1], Proposition 4.2). For an irreducible Wieler solenoid $(X, f)$, there is a Markov partition $\mathcal{P}$ such that, for every $x \in R \in \mathcal{P}$ and $0<\epsilon \leq \epsilon_{0}, X^{s}(x, R)$ is clopen in $X^{s}\left(x, \epsilon_{0}\right)$. 
Let $(\Sigma, \sigma)$ be the SFT associated with the Markov partition $\mathcal{P}$ as above. Then there is a bijection between the alphabet of $(\Sigma, \sigma)$ and $\mathcal{P}$ given by $a \mapsto R_{a}$. Nekrashevych ([7], Lemma 4.4) showed that the map $\pi: \Sigma \rightarrow X$ given by

$$
\cdots a_{-2} a_{-1} \cdot a_{0} a_{1} \cdots \mapsto \bigcap_{n \in \mathbb{Z}} f^{n}\left(R_{a_{n}}\right)
$$

is an s-resolving factor map. For each $x \in X$, we call the elements of $\pi^{-1}(x)$ codes of $x$.

Lemma 3. ([7], Lemma 4.3] If $x, y \in R_{a_{0}}$ satisfy $\pi\left(\cdots a_{-2} a_{-1} \cdot a_{0} a_{1} \cdots\right)=x$ and $\pi\left(\cdots b_{-2} b_{-1} \cdot b_{0} b_{1} \cdots\right)=$ $y$ with $a_{0}=b_{0}$, then $\pi\left(\cdots a_{-2} a_{-1} \cdot b_{0} b_{1} \cdots\right)=[x, y]$. In particular, the set $X^{s}\left(x, R_{a_{0}}\right)$ coincides with the set of points having codes of the form $\cdots a_{-2} a_{-1} \cdot a_{0} s_{1} s_{2} \cdots$.

\section{Adjacency Semigroups}

Let us fix an irreducible Wieler solenoid $(X, f)$ and a Markov partition $\mathcal{P}=\left\{R_{a}\right\}_{a \in \mathcal{A}}$ as given in Lemma 2 with its associated SFTs $\left(\Sigma^{+}, \sigma^{+}\right)$and $(\Sigma, \sigma)$, whose alphabets are $\mathcal{A}$, and an $s$-resolving factor map $\pi: \Sigma \rightarrow X$.

We observe that if $R_{a_{0}} \cap R_{b_{0}} \neq \varnothing$ and $x \in R_{a_{0}} \cap R_{b_{0}}$, then $x$ has two distinct codes $\cdots a_{-2} a_{-1} \cdot a_{0} a_{1} \cdots$ and $\cdots b_{-2} b_{-1} \cdot b_{0} b_{1} \cdots$ in $\pi^{-1}(x)$. Now we define a partial transformation $T_{\left(a_{0} ; b_{0}\right)}$ of $\Sigma^{+}$as follows:

$$
T_{\left(a_{0} ; b_{0}\right)}: a_{0} s_{1} s_{2} \cdots \mapsto b_{0} t_{1} t_{2} \cdots
$$

if and only if

$$
\pi\left(\cdots a_{-2} a_{-1} \cdot a_{0} s_{1} s_{2} \cdots\right)=\pi\left(\cdots b_{-2} b_{-1} \cdot b_{0} t_{1} t_{2} \cdots\right) .
$$

Lemma 4 ([7], Proposition 4.5). The transformation $T_{\left(a_{0} ; b_{0}\right)}$ is a well-defined partial homeomorphism of $\Sigma^{+}$ depending only on the letters $a_{0}$ and $b_{0}$.

Definition 7 ([7], Definition 4.2). The adjacency semigroup of an irreducible Wieler solenoid $(X, f)$ is the inverse semigroup generated by the set of partial homeomorphisms $\left\{\operatorname{Id}_{\Sigma^{+}}, T_{\left(a_{0} ; b_{0}\right)}: R_{a_{0}} \cap R_{b_{0}} \neq \varnothing\right\}$ of the one-sided $\operatorname{SFT}\left(\Sigma^{+}, \sigma^{+}\right)$.

Theorem 3 ([7], Theorem 4.6). The adjacency semigroup $H$ of an irreducible Wieler solenoid $(X, f)$ is a contracting self-similar inverse semigroup. Moreover, its limit solenoid $\left(S_{H}, \sigma\right)$ is topologically conjugate to the Wieler solenoid $(X, f)$.

Remark 1. When we have

$$
\pi\left(\cdots a_{-1} \cdot a_{0} \cdots a_{n} s_{n+1} s_{n+2} \cdots\right)=\pi\left(\cdots b_{-1} \cdot b_{0} \cdots b_{n} t_{n+1} t_{n+2} \cdots\right)
$$

so that

$$
T_{\left(a_{0} ; b_{0}\right)}\left(a_{0} \cdots a_{n} s_{n+1} s_{n+2} \cdots\right)=b_{0} \cdots b_{n} t_{n+1} t_{n+2} \cdots,
$$

there is a finite set $\left\{h_{1}, \ldots, h_{k}\right\} \subset H$ such that the sets $\operatorname{Dom} h_{i}$ are disjoint, $\cup a_{0} \cdots a_{n} \operatorname{Dom} h_{i} \subset \operatorname{Dom} T_{\left(a_{0} ; b_{0}\right)}$ and

$$
T_{\left(a_{0} ; b_{0}\right)}\left(a_{0} \cdots a_{n} w\right)=b_{0} \cdots b_{n} h_{i}(w)
$$

for every $w \in \operatorname{Dom} h_{i}$. We will denote $T_{\left(a_{0} ; b_{0}\right)} \mid \cup a_{0} \cdots a_{n} \operatorname{Dom} h_{i}$ as $T_{\left(a_{0} \cdots a_{n} ; b_{0} \cdots b_{n}\right)}$.

Let us consider the inverse semigroup $H^{\prime}$ generated by

$$
\left\{\operatorname{Id}_{\Sigma^{+}}, T_{\left(a_{0} \cdots a_{n} ; b_{0} \cdots b_{n}\right)}: R_{a_{0}} \cap R_{b_{0}} \neq \varnothing\right\}
$$

acting on $\Sigma^{+}$. Even though $H^{\prime}$ contains $H$ as a subsemigroup,

$$
T_{\left(a_{0} \cdots a_{n} ; b_{0} \cdots b_{n}\right)}=T_{\left(a_{0} ; b_{0}\right)} \mid \cup a_{0} \cdots a_{n} \operatorname{Dom} h_{i}
$$


implies that $H^{\prime}$ is also a contracting self-similar inverse semigroup whose nucleus is the same as that of $H$ and that $H^{\prime}$ action on $\Sigma^{+}$coincides with the action of $H$. Hence, the asymptotic equivalence relations on $\Sigma$ given by $H^{\prime}$ and $H$ concur with each other. and so do the limit solenoids $S_{H^{\prime}}$ and $S_{H}$. From now on, we will use $H^{\prime}$ as the adjacency semigroup of an irreducible Wieler solenoid, and denote it simply as $H$ instead of $H^{\prime}$.

Remark 2. In the proof of ([7], Theorem 4.6), Nekrashevych showed that the s-resolving factor map $\pi: \Sigma \rightarrow$ $X \simeq S_{H}$ is equal to the quotient map $q: \Sigma \rightarrow S_{H}$.

Comparing with limit solenoids of self-similar groups (see $[19,20]$ for self-similar groups), it is easy to obtain the following property of unstable equivalence relation on $\left(S_{H}, \sigma\right)$.

Proposition 1 ([20], Proposition 6.8). Suppose that $H$ is the adjacency semigroup of an irreducible Wieler solenoid with the nucleus $\mathcal{N}$ and that $\left(S_{H}, \sigma\right)$ is the limit solenoid of $H$. For $x, y \in S_{H}$, let

$$
\cdots a_{-1} \cdot a_{0} a_{1} \cdots \in q^{-1}(x) \text { and } \cdots b_{-1} \cdot b_{0} b_{1} \cdots \in q^{-1}(y) \text {, }
$$

where $q: \Sigma \rightarrow S_{H}$ is the quotient map. Then $x$ is unstably equivalent to $y$ if and only if there exist $n \in \mathbb{Z}$ and $h \in \mathcal{N}$ such that

$$
h\left(a_{n} a_{n+1} \cdots\right)=b_{n} b_{n+1} \cdots
$$

\section{Groupoid Equivalence}

We show that for a Wieler solenoid $(X, f)$ and it adjacency semigroup $H$, the groupoid of germs of $H$, the tight groupoid of $H$ and the unstable groupoid of $(X, f)$ are equivalent to each other.

Theorem 4. Suppose that $H$ is the adjacency semigroup of an irreducible Wieler solenoid and that $\left(S_{H}, \sigma\right)$ is the limit solenoid of $H$. Then the groupoid of germs $\Gamma$ of $H$ is equivalent to the unstable groupoid $G_{u}$ of $\left(S_{H}, \sigma\right)$ in the sense of Muhly, Renault and Williams.

Proof. We imitate the proof of ([6], Theorem 4.8) to construct a $\left(G_{u}, \Gamma\right)$-equivalence $Z$ of Muhly, Renault and Williams [21]. Let

$$
Z=\left\{(x, v) \in S_{H} \times \Sigma^{+}: \exists \cdots a_{-1} . v \in \Sigma \text { such that } \pi\left(\cdots a_{-1} \cdot v\right) \sim_{u} x\right\}
$$

where $\sim_{u}$ is the unstable equivalence. For each $h \in H$, let

$$
Z_{h}=\left\{(x, v) \in S_{H} \times \Sigma^{+}: \exists \cdots b_{-1} \cdot h(v) \in \Sigma \text { such that } \pi\left(\cdots b_{-1} \cdot h(v)\right)=x\right\} .
$$

It is easy to obtain $Z=\bigcup_{h \in H} Z_{h}$ from Proposition 1. On $Z_{h}$, we give the subspace topology from $S_{H} \times \Sigma^{+}$, and $Z$ has the direct limit topology from the union. We show that $Z_{h}$ is closed in $S_{H} \times \Sigma^{+}$. Then $Z_{h}$ is a locally compact Hausdorff space under the subspace topology, and it is trivial that $Z$ is also a locally compact Hausdorff space.

Let $p: \Sigma \rightarrow \Sigma^{+}$be the canonical projection given by $\cdots a_{-1} \cdot a_{0} a_{1} \cdots \mapsto a_{0} a_{1} \cdots$. Then $p$ is continuous, and

$$
\pi \times\left(h^{*} \circ p\right): \Sigma \rightarrow S_{H} \times \Sigma^{+} \text {given by } w \mapsto\left(\pi(w), h^{*} \circ p(w)\right)
$$

is also continuous. Here we use the fact that $\pi(w)=q(w)$, see Remark 2. It is easy to check that $Z_{h}=\pi \times\left(h^{*} \circ p\right)(\Sigma)$, and $Z_{h}$ is closed in $S_{H} \times \Sigma^{+}$. The Hausdorff property is trivial.

The left action of $G_{u}$ is

$$
(y, x) \cdot(x, v)=(y, v)
$$


and the right action of $\Gamma$ on $\mathrm{Z}$ is

$$
(x, v) \cdot[h, v]=(x, h(v)) .
$$

Then it is routine to verify that $Z$ is a left principal $G_{u}$-space, a right principal $\Gamma$-space, and that the $G_{u}$ and $\Gamma$ actions are commuting.

We recall that each $Z_{h}$ is canonically included in $Z$. Define

$$
\begin{aligned}
& \rho: Z \rightarrow G_{u}^{0} \simeq S_{H} \text { by }(x, v) \mapsto x \text { and } \\
& \tau: Z \rightarrow \Gamma^{0} \simeq \Sigma^{+} \text {by }(x, v) \mapsto v .
\end{aligned}
$$

Since each $Z_{h}$ has the subspace topology from $S_{H} \times \Sigma^{+}$, the canonical projections $Z_{h} \rightarrow S_{H}$ and $Z_{h} \rightarrow \Sigma^{+}$given by $(x, v) \mapsto x$ and $(x, v) \mapsto v$, respectively, are continuous open maps. Then the universal property of the direct limit topology on $Z$ implies that $\rho$ and $\tau$ are continuous open maps.

For the left $G_{u}$-action and the right $\Gamma$-action on $Z$, corresponding orbits of an $(x, v) \in Z$ are

$$
\begin{aligned}
G_{u} \cdot(x, v) & =\left\{(y, v): x \sim_{u} y\right\} \text { and } \\
(x, v) \cdot \Gamma & =\{(x, h(v)): h \in H \text { and } v \in \operatorname{Dom} h\} .
\end{aligned}
$$

So the maps $\rho$ and $\tau$ induce bijections of $Z / \Gamma$ onto $G_{u}^{0}$ and $G_{u} \backslash Z$ onto $\Gamma^{0}$, respectively. Therefore $Z$ is an $\left(G_{u}, \Gamma\right)$-equivalence, and $G_{u}$ is equivalent to $\Gamma$ in the sense of Muhly, Renault and Williams.

Remark 3. We remind that the unstable groupoid $G_{u}$ of $\left(S_{H}, \sigma\right)$ is not an étale groupoid, but the groupoid of germs $\Gamma$ of $H$ is étale. In [12], they obtained an étale groupoid by restricting to an abstract transversal that is equivalent to $G_{u}$.

\section{Tight Groupoids}

We show that the groupoid of germs and the tight groupoid of an adjacency semigroup are equivalent to each other.

We omit the proof of the following lemma as it is trivial from the definition of idempotents and the compactness of $\Sigma^{+}$.

Lemma 5. Suppose that $H$ is the adjacency semigroup of an irreducible Wieler solenoid acting on $\Sigma^{+}$and that $E$ is the set of idempotents of $H$. Then

1. $\left\{\left.\operatorname{Id}\right|_{\operatorname{Dom} T_{(\alpha ; \beta)}}: \alpha, \beta \in \mathcal{B}_{n}\left(\Sigma^{+}\right), T_{(\alpha ; \beta)} \neq 0\right\}$ generates $E$.

2. For $\mu, v \in E, \mu \leq v$ if and only if $\operatorname{Dom} \mu \subset \operatorname{Dom} v$.

3. For $\mu \in E, \operatorname{Dom} \mu$ is a clopen subset of $\Sigma^{+}$.

Remark 4. For each $\alpha \in \mathcal{B}_{n}\left(\Sigma^{+}\right)$, there is at least one $\beta \in \mathcal{B}_{n}\left(\Sigma^{+}\right)$such that $T_{(\alpha ; \beta)} \neq 0$, say $\beta=\alpha$.

Proposition 2. For each $\alpha, \beta \in \mathcal{B}_{n}\left(\Sigma^{+}\right)$such that $T_{(\alpha ; \beta)} \neq 0$, let

$$
D(\alpha ; \beta)=\left\{\mu \in E: \operatorname{Dom} T_{(\alpha ; \beta)} \subset \operatorname{Dom} \mu\right\} .
$$

Then $D(\alpha ; \beta)$ is a filter.

Proof. It is trivial that $D(\alpha ; \beta) \neq \varnothing$ as $\left.I d\right|_{\operatorname{Dom} T_{(\alpha ; \beta)}}=T_{(\alpha ; \beta)}^{*} T_{(\alpha ; \beta)} \in D(\alpha ; \beta)$. If $\mu \in D(\alpha ; \beta)$ and $\mu \leq v$, then Lemma 5 implies

$$
\operatorname{Dom} T_{(\alpha ; \beta)} \subset \operatorname{Dom} \mu \subset \operatorname{Dom} v
$$

so that $v \in D(\alpha ; \beta)$. For any $\mu, v \in D(\alpha ; \beta)$,

$$
\operatorname{Dom} T_{(\alpha ; \beta)} \subset \operatorname{Dom} \mu \text { and } \operatorname{Dom} T_{(\alpha ; \beta)} \subset \operatorname{Dom} v
$$


mean that $T_{(\alpha ; \beta)}^{*} T_{(\alpha ; \beta)} \leq \mu, \nu$. Hence $D(\alpha ; \beta)$ is a filter.

Lemma 6. For $\alpha, \beta \in \mathcal{B}_{n}\left(\Sigma^{+}\right)$and $\alpha a_{n}, \beta b_{n} \in \mathcal{B}_{n+1}\left(\Sigma^{+}\right)$such that $T_{(\alpha ; \beta)}$ and $T_{\left(\alpha a_{n} ; \beta b_{n}\right)}$ are nonzero maps, $D(\alpha ; \beta) \subset D\left(\alpha a_{n} ; \beta b_{n}\right)$.

Proof. If $\mu$ is an element of $D(\alpha ; \beta)$, Definition 4 implies

$$
\operatorname{Dom} \mu \supset \operatorname{Dom} T_{(\alpha ; \beta)}=\cup \alpha \operatorname{Dom} h_{i} \supset \cup \alpha a_{n} \operatorname{Dom} g_{j} \supset \operatorname{Dom} T_{\left(\alpha a_{n} ; \beta b_{n}\right)} .
$$

Thus $\mu$ is an element of $D\left(\alpha a_{n} ; \beta b_{n}\right)$, and we have $D(\alpha ; \beta) \subset D\left(\alpha a_{n} ; \beta b_{n}\right)$.

Lemma 7. For each $u=a_{0} a_{1} \cdots \in \Sigma^{+}$and any $b_{0} b_{1} \cdots \in \Sigma^{+}$such that $T_{\left(a_{0} ; b_{0}\right)}\left(a_{0} a_{1} \cdots\right)=b_{0} b_{1} \cdots$, let $D(u)=\bigcup_{n=0}^{\infty} D\left(a_{0} \cdots a_{n} ; b_{0} \cdots b_{n}\right)$. Then we have $D(u)=\{\mu \in E: u \in \operatorname{Dom} \mu\}$.

Proof. Let $\mu \in \bigcup_{n=0}^{\infty} D\left(a_{0} \cdots a_{n} ; b_{0} \cdots b_{n}\right)$. Then there is a nonnegative integer $n$ such that $\mu \in$ $D\left(a_{0} \cdots a_{n} ; b_{0} \cdots b_{n}\right)$. So $u \in \operatorname{Dom} T_{\left(a_{0} \cdots a_{n} ; b_{0} \cdots b_{n}\right)} \subset \operatorname{Dom} \mu$.

On the other hand, if $u \in \operatorname{Dom} \mu$, then $u$ is an interior point of Dom $\mu$ by Lemma 5 , and there is a nonnegative integer $n$ such that

$$
u \in C_{0}\left(a_{0} \cdots a_{n}\right) \subset \operatorname{Dom} \mu .
$$

Since Dom $T_{\left(a_{0} \cdots a_{n} ; b_{0} \cdots b_{n}\right)}=\cup a_{0} \cdots a_{n} \operatorname{Dom} h_{i}$ by Definition 4 , we have

$$
\operatorname{Dom} T_{\left(a_{0} \cdots a_{n} ; b_{0} \cdots b_{n}\right)} \subset C_{0}\left(a_{0} \cdots a_{n}\right) \subset \operatorname{Dom} \mu
$$

and $\mu \in D\left(a_{0} \cdots a_{n} ; b_{0} \cdots b_{n}\right)$.

Proposition 3. For each $u=a_{0} a_{1} \cdots \in \Sigma^{+}, D(u)$ is an ultrafilter.

Proof. By Lemma 7, $D(u)$ is a filter. If $D(u)$ is not an ultrafilter, then there is a filter $C$ such that $D(u) \subsetneq C$. Choose an idempotent $\mu \in C \backslash D(u)$, i.e., $u \notin \operatorname{Dom} \mu$.

Because $\mu$ and $\left.\operatorname{Id}\right|_{\operatorname{Dom} T_{\left(a_{0} \cdots a_{n} ; b_{0} \cdots b_{n}\right)}}$ are elements of $C$ for every nonnegative integer $n$, there is a

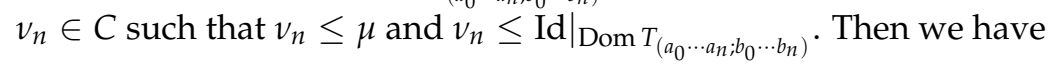

$$
\operatorname{Dom} v_{n} \subset \operatorname{Dom} \mu \cap \operatorname{Dom} T_{\left(a_{0} \cdots a_{n} ; b_{0} \cdots b_{n}\right)} \text {. }
$$

So we have $\operatorname{Dom} \mu \cap \operatorname{Dom} T_{\left(a_{0} \cdots a_{n} ; b_{0} \cdots b_{n}\right)} \neq \varnothing$ for every nonnegative integer $n$ and

$$
\operatorname{Dom} \mu \cap \operatorname{Dom} T_{\left(a_{0} \cdots b_{n} ; b_{0} \cdots b_{n}\right)} \supset \operatorname{Dom} \mu \cap \operatorname{Dom} T_{\left(a_{0} \cdots a_{n+1} ; b_{0} \cdots b_{n+1}\right)} \text {. }
$$

Since the domain of every idempotent is a compact set by Lemma 5, the Cantor Intersection Theorem implies

$$
\begin{aligned}
\bigcap \operatorname{Dom} \mu \cap \operatorname{Dom} T_{\left(a_{0} \cdots a_{n} ; b_{0} \cdots b_{n}\right)} & =\operatorname{Dom} \mu \cap \bigcap \operatorname{Dom} T_{\left(a_{0} \cdots a_{n} ; b_{0} \cdots b_{n}\right)} \\
& =\operatorname{Dom} \mu \cap\{u\} \\
& \neq \varnothing .
\end{aligned}
$$

Thus we have $u \in \operatorname{Dom} \mu$, a contradiction. Therefore $D(u)$ is an ultrafilter.

Proof of the following property is exactly the same as that of ([22], Lemma 4.13), but we include its proof here for completeness. 
Proposition 4 ([22], Lemma 4.13). Suppose that $H$ is the adjacency semigroup of an irreducible Wieler solenoid acting on $\Sigma^{+}$and that $E$ is the set of idempotents of $H$. Then every ultrafilter in $E$ is given as $D(u)$ for some $u \in \Sigma^{+}$.

Proof. Let $D$ be an ultrafilter and consider a collection

$$
C=\{\operatorname{Dom} \mu: \mu \in D\} .
$$

Then each Dom $\mu$ is a compact subset of $\Sigma^{+}$by Lemma 5 , and $\Sigma^{+}$is also a compact space. For any finite collection $\mu_{1}, \ldots, \mu_{n} \in D$, the third condition of filters implies that there is an idempotent $v \in D$ such that $v \leq \mu_{i}$ for every $i=1, \ldots, n$. So, we have

$$
\varnothing \neq \operatorname{Dom} v \subset \cap_{i=1}^{n} \operatorname{Dom} \mu_{i}
$$

and the collection $C$ has the finite intersection property. Hence the intersection

$$
\bigcap_{\mu \in D} \operatorname{Dom} \mu
$$

is nonempty as $\Sigma^{+}$is a compact space. We choose an infinite path $u \in \cap_{\mu \in D}$ Dom $\mu$, and consider its corresponding ultrafilter $D(u)$ constructed in Lemma 7. For every $\mu \in D, u \in \operatorname{Dom} \mu$ implies $\mu \in D(u)$ and $D \subset D(u)$. Since $D$ is an ultrafilter, we conclude $D=D(u)$.

Now the following lemma is trivial by Lemma 1 and Proposition 4.

Lemma 8. Let $\Phi: \Sigma^{+} \rightarrow \hat{E}_{\infty}$ be given by $u \mapsto \phi_{u}$ where $\phi_{u}: E \rightarrow\{0,1\}$ is

$$
\phi_{u}(\mu)= \begin{cases}1 & \text { if } \mu \in D(u) \\ 0 & \text { otherwise }\end{cases}
$$

Then $\Phi$ is a bijection.

Again, the proof of the following proposition is exactly the same as that of ([22], Proposition 4.14), but we include it for completeness.

Proposition 5. The bijection $\Phi: \Sigma^{+} \rightarrow \hat{E}_{\infty}$ is a homeomorphism. Moreover, $\hat{E}_{\infty}$ coincides with $\hat{E}_{\text {tight }}$.

Proof. Because $\Sigma^{+}$is a compact space, we only need to show that $\Phi$ is a continuous map. For every $\mu \in E$ and $\phi_{u} \in \hat{E}_{\infty}$,

$$
D_{\mu}=\left\{\phi_{u} \in \hat{E}_{\infty}: \phi_{u}(\mu)=1\right\} \text { and } \Delta_{\mu}=\left\{\phi_{u} \in \hat{E}_{\infty}: \phi_{u}(\mu)=0\right\}
$$

are a subbasis for the subspace topology on $\hat{E}_{\infty}$. By Lemma 8 , we can rewrite $D_{\mu}$ and $\Delta_{\mu}$ as

$$
D_{\mu}=\left\{\phi_{u} \in \hat{E}_{\infty}: u \in \operatorname{Dom} \mu\right\} \text { and } \Delta_{\mu}=\left\{\phi_{u} \in \hat{E}_{\infty}: u \notin \operatorname{Dom} \mu\right\} .
$$

So we have

$$
\begin{aligned}
& \Phi^{-1}\left(D_{\mu}\right)=\left\{u \in \Sigma^{+}: u \in \operatorname{Dom} \mu\right\}=\operatorname{Dom} \mu \text { and } \\
& \Phi^{-1}\left(\Delta_{\mu}\right)=\left\{u \in \Sigma^{+}: u \notin \operatorname{Dom} \mu\right\}=(\operatorname{Dom} \mu)^{c} .
\end{aligned}
$$

Then $\operatorname{Dom} \mu$ is a clopen set implies that $\phi$ is continuous so that $\Phi$ is a homeomorphism. 
Since $\Sigma^{+}$is a compact space and $\phi$ is a homeomorphism, $\hat{E}_{\infty}$ is also a compact subspace of $\hat{E}_{0}$. Thus we have $\hat{E}_{\infty}=\overline{\hat{E}}_{\infty}=\hat{E}_{\text {tight }}$.

Remark 5. If $E$ is a Boolean algebra, then Stone duality implies $\hat{E}_{\infty}=\hat{E}_{\text {tight }}$ ([23], p. 815).

We remind that the groupoid of germs $\Gamma$ of $H$ and the tight groupoid $\mathcal{G}_{\text {tight }}$ of $H$ are induced from $H$-actions on $\Sigma^{+}$and $\hat{E}_{\text {tight }}=\hat{E}_{\infty}$, respectively. Then the $H$-action on $\Sigma^{+}$can be transferred to an action on $\hat{E}_{\text {tight }}$ via $\Phi: \Sigma^{+} \rightarrow \hat{E}_{\text {tight }}$.

The action of $H$ on $\hat{E}_{\text {tight }}$, denoted $\theta$, was given as follows: For each idempotent $\mu \in E$ with

$$
D_{\mu}=\left\{\phi_{u} \in \hat{E}_{\text {tight }}: \phi_{u}(\mu)=1\right\}
$$

and for every $h \in H, \theta_{h}: D_{h^{*} h} \rightarrow D_{h h^{*}}$ is defined by $\phi_{u} \mapsto \theta_{h}\left(\phi_{u}\right)$ such that $\theta_{h}\left(\phi_{u}\right)(v)=\phi_{u}\left(h^{*} v h\right)$ for every $v \in E$.

Theorem 5. Suppose that $H$ is the adjacency semigroup of an irreducible Wieler solenoid. Then the groupoid of germs $\Gamma$ of $H$ is isomorphic to the tight groupoid $\mathcal{G}_{\text {tight }}$ of $H$.

Proof. To obtain a groupoid isomorphism between $\Gamma$ and $\mathcal{G}_{\text {tight }}$, we only need to show that transfer of $H$-action on $\Sigma^{+}$by the homeomorphism $\Phi$ commutes with $H$-action on $\hat{E}_{\text {tight }}$ ([8], Proposition 1$)$, i.e., prove

$$
\Phi(h(u))=\theta_{h}(\Phi(u))
$$

for every $h \in H$ and $u \in \operatorname{Dom} h$.

Before proving the above equality, we remind that every idempotent is an identity map on an open subset of $\Sigma^{+}$(see Example 1) and that, for $h \in H$ and $u \in \operatorname{Dom} h, h(u) \in \operatorname{Dom} h^{*}$.

For every idempotent $v \in E$, we have

$$
\Phi(h(u))(v)=\phi_{h(u)}(v)= \begin{cases}1 & \text { if } h(u) \in \operatorname{Dom} v \\ 0 & \text { if } h(u) \notin \operatorname{Dom} v\end{cases}
$$

and

$$
\theta_{h}(\Phi(u))(v)=\theta_{h}\left(\phi_{u}\right)(v)=\phi_{u}\left(h^{*} v h\right)= \begin{cases}1 & \text { if } u \in \operatorname{Dom} h^{*} v h \\ 0 & \text { if } u \notin \operatorname{Dom} h^{*} v h\end{cases}
$$

If $\Phi(h(u))(v)=1$, then $h(u)$ is an element of Dom $v$ and

$$
v(h(u))=h(u) \in \operatorname{Dom} h^{*} .
$$

So we have $u \in \operatorname{Dom} h^{*} v h$ and

$$
\theta_{h}(\Phi(u))(v)=1 .
$$

On the other hand, if $\theta_{h}(\Phi(u))(v)=1$, then we have $u \in \operatorname{Dom} h^{*} v h$. Thus $h(u)$ is included in Dom $v$ and

$$
\Phi(h(u))(v)=1 .
$$

Hence, $\Phi(h(u))(v)=1$ if and only if $\theta_{h}(\Phi(u))(v)=1$ for every $v \in E$, which implies $\Phi(h(u))=$ $\theta_{h}(\Phi(u))$ for every $h \in H$ and $u \in \operatorname{Dom} h$. Therefore, $\Gamma$ is isomorphic to $\mathcal{G}_{\text {tight }}$.

Then next properties are obvious from Theorems 4 and 5 .

Theorem 6. Suppose that $H$ is the adjacency semigroup of an irreducible Wieler solenoid and that $\left(S_{H}, \sigma\right)$ is the limit solenoid of $H$. Then the tight groupoid of $H$ is equivalent to the unstable groupoid of $\left(S_{H}, \sigma\right)$ in the sense of Muhly, Renault and Williams. 
Now, strong Morita equivalence is a trivial consequence of groupoid equivalence.

Theorem 7. Suppose that $H$ is the adjacency semigroup of an irreducible Wieler solenoid, that $\Gamma$ and $\mathcal{G}_{\text {tight }}$ are the groupoid of germs of $H$ and the tight groupoid of $H$, respectively, and that $G_{u}$ is the unstable groupoid of the Wieler solenoid. Then the groupoid $C^{*}$-algebras $C^{*}(\Gamma), C^{*}\left(\mathcal{G}_{\text {tight }}\right)$ and the unstable algebra $C^{*}\left(G_{u}\right)$ are strongly Morita equivalent to each other.

\section{Traces and Invariant Means}

We consider the uniqueness of tracial state on the groupoid of germs algebra $C^{*}(\Gamma)$ of an irreducible Wieler solenoid $(X, f)$. Every material in this section is taken from [23].

Let $S$ be an inverse semigroup and $E(S)$ the set of all idempotents in $S$. For $s, t \in S$, we say that $s$ and $t$ are compatible if $s^{*} t, s t^{*} \in E(S)$, and a set $F \subset S$ is called compatible if every pair of elements of $F$ is compatible. For $s, t \in S$, the join (or least upper bound) of $s$ and $t$ is denoted $s \vee t$ if it exists. We call $S$ distributive if whenever we have a finite compatible set $F \subset S$, then $\vee_{s \in F} S$ exists in $S$ and for every $t \in S$ we have

$$
t\left(\vee_{s \in F} s\right)=\vee_{s \in F} t s \text { and }\left(\vee_{s \in F} s\right) t=\vee_{s \in F} s t .
$$

Definition 8 ([23], Definition 2.2). A Boolean inverse monoid is a distributive inverse monoid $S$ with the property that $E(S)$ is a Boolean algebra.

For an étale groupoid $(\mathcal{G}, d, r)$, an open set $U \subset \mathcal{G}$ is called a bisection if $\left.r\right|_{U}$ and $\left.d\right|_{U}$ are injective. An étale groupoid $\mathcal{G}$ is called ample if the set of compact bisections forms a basis for the topology on $\mathcal{G}$. The set of compact bisections, denoted $\mathcal{G}^{a}$ and called the ample semigroup of $\mathcal{G}$, is a distributive inverse semigroup.

Lemma 9 ([23]). Suppose that $H$ is the adjacency semigroup of an irreducible Wieler solenoid and that $\Gamma$ is the groupoid of germs of $H$. Then

1. $\Gamma$ is an ample groupoid,

2. $\Gamma^{a}$ is a Boolean inverse monoid, and

3. $\Gamma \simeq \mathcal{G}_{\text {tight }}\left(\Gamma^{a}\right)$.

Proof. The first two properties are from the fact that $\Sigma^{+}$is a Cantor set. The third property is from ([23], p. 816).

Remark 6. It is not difficult to observe that every bisection in $\Gamma$ is given as $O(h, U)=\{[h, u]: u \in U\}$ where $h$ is an element of $H$ and $U$ is any open subset of Dom $h^{*} h$. Since $H$ is generated by $\left\{T_{(\alpha ; \beta)}: \alpha, \beta \in\right.$ $\left.\mathcal{B}_{n}\left(\Sigma^{+}\right), n \geq 0\right\}$ and the collection of all cylinder sets is a basis for the topology on $\Sigma^{+}=\Gamma^{(0)}, \Gamma^{a}$ is generated by $O(h, V)$ such that $h=T_{(\alpha ; \beta)}$ and $V=C_{0}(\alpha \gamma) \subset \operatorname{Dom} h$. See [24] for the structure of ample semigroups.

Lemma 10. The groupoid of germs $\Gamma$ of $H$ is an étale amenable principal Hausdorff groupoid.

Proof. Étale property is trivial by construction of $\Gamma$. By ([12], Theorem 1.1) the unstable groupoid $G_{u}$ is an amenable groupoid. So $\Gamma$ is amenable by Theorem 4 and ([25], Theorem 2.2.17).

To show that $\Gamma$ is a principal groupoid, for $[h, u] \in \Gamma$ we need to check $u=h(u)$ if and only if $h$ is an idempotent.

If $h \in H$ is an idempotent, then $h$ is an identity map on an open subset of $\Sigma^{+}$, so $u=h(u)$ holds for every $u \in \operatorname{Dom} h$. For the other direction, we only consider $T_{(\alpha ; \beta)}$ as $H$ is generated by $T_{(\alpha ; \beta)}$. Then $u \in \operatorname{Dom} T_{(\alpha ; \beta)}$ and $T_{(\alpha ; \beta)}(u)=u$ mean that $u=\alpha v$ for some $v \in \Sigma^{+}$and $\beta=\alpha$. So we have $T_{(\alpha ; \beta)}=T_{(\alpha ; \alpha)}$ which is the identity map on the cylinder set $C_{0}(\alpha)$. Hence $T_{(\alpha ; \beta)}$ is an idempotent, and $\Gamma$ is a principal groupoid. 
Because $\Gamma$ is an étale groupoid and $\Gamma^{(0)}$ is homeomorphic to $\Sigma^{+}$, Hausdorff property is obvious by ([15], Proposition 3.10).

Definition 9. Let $S$ be a Boolean inverse monoid. A nonzero function $m: E(S) \rightarrow[0, \infty)$ is called an invariant mean if

1. $m\left(s^{*} s\right)=m\left(s s^{*}\right)$ for every $S \in S$

2. $\quad m(\mu \vee v)=m(\mu)+m(v)$ for all $\mu, v \in E(S)$ such that $\mu v=0$.

If in addition $m(1)=1$, we call $m$ a normalized invaraint mean. We denote $M(S)$ by the affine space of all normalized invariant means on $S$.

Definition 10. Let $A$ be a $C^{*}$-algebra. A bounded linear functional $\tau: A \rightarrow \mathbb{C}$ is called a trace if

1. $\tau\left(a^{*} a\right) \geq 0$ for every $a \in A$

2. $\tau(a b)=\tau(b a)$ for all $a, b \in A$.

A trace $\tau$ on a unital $C^{*}$-algebra is called a tracial state if $\tau(1)=1$. The set of all tracial states of a $C^{*}$-algebra $A$ is denoted $T(A)$.

Theorem 8 ([23], Theorem 4.13). Let $S$ be a Boolean inverse monoid such that $\mathcal{G}_{\text {tight }}(S)$ is an amenable principal Hausdorff groupoid. Then

$$
T\left(C^{*}\left(\mathcal{G}_{\text {tight }}(S)\right)\right) \simeq M(S) .
$$

By Lemmas 9 and 10, $\Gamma^{a}$ is a Boolean inverse monoid such that $\mathcal{G}_{\text {tight }}\left(\Gamma^{a}\right)$ is an amenable principal Hausdorff groupoid. Therefore we have the following:

Proposition 6. Let $\Gamma$ be the groupoid of germs of the adjacency semigroup of a Wieler solenoid. Then

$$
T\left(C^{*}(\Gamma)\right) \simeq T\left(C^{*}\left(\mathcal{G}_{\text {tight }}\left(\Gamma^{a}\right)\right)\right) \simeq M\left(\Gamma^{a}\right) .
$$

To figure out $M\left(\Gamma^{a}\right)$, we only consider $O\left(T_{(\alpha ; \beta)}, C_{0}(\alpha \gamma)\right) \in \Gamma^{a}$ where $\gamma$ and $\alpha \gamma$ are finite allowed words in $\Sigma^{+}$(see Remark 6). We observe

$$
O\left(T_{(\alpha ; \beta)}, C_{0}(\alpha \gamma)\right)^{*}=O\left(T_{(\beta ; \alpha)}, C_{0}\left(T_{(\alpha ; \beta)}(\alpha \gamma)\right)\right)
$$

such that $T_{(\alpha ; \beta)}(\alpha \gamma)=\beta \delta$ with $|\alpha \gamma|=|\beta \delta|$ and

$$
\begin{aligned}
& O\left(T_{(\alpha ; \beta)}, C_{0}(\alpha \gamma)\right) O\left(T_{(\alpha ; \beta)}, C_{0}(\alpha \gamma)\right)^{*}=O\left(\left.\operatorname{Id}\right|_{\operatorname{Dom} T_{(\beta ; \alpha)}}, C_{0}(\beta \delta)\right) \\
& O\left(T_{(\alpha ; \beta)}, C_{0}(\alpha \gamma)\right)^{*} O\left(T_{(\alpha ; \beta)}, C_{0}(\alpha \gamma)\right)=O\left(\left.\operatorname{Id}\right|_{\operatorname{Dom} T_{(\alpha ; \beta)}}, C_{0}(\alpha \gamma)\right) .
\end{aligned}
$$

Here we note that $\left.\left.\mathrm{Id}\right|_{\Sigma^{+}} \operatorname{Id}\right|_{\operatorname{Dom} T_{(\alpha ; \beta)}}=\left.\left.\mathrm{Id}\right|_{\operatorname{Dom} T_{(\alpha ; \beta)}} \operatorname{Id}\right|_{\operatorname{Dom} T_{(\alpha ; \beta)}}$ and the definition of the groupoid of germs imply that it is unnecessary to restrict the domain of Id map inside bisection.

If $\mu$ is a normalized invariant mean on $\Gamma^{a}$, then we have

$$
\mu\left(O\left(\operatorname{Id}, C_{0}(\alpha \gamma)\right)\right)=\mu\left(O\left(\operatorname{Id}, C_{0}(\beta \delta)\right)\right) .
$$

So we obtain that for all $\eta, \zeta \in \mathcal{B}_{n}\left(\Sigma^{+}\right)$

$$
\mu\left(O\left(\operatorname{Id}, C_{0}(\eta)\right)\right)=\mu\left(O\left(I d, C_{0}(\zeta)\right)\right) .
$$

In addition, the set $\left\{C_{0}(\eta): \eta \in \mathcal{B}_{n}\left(\Sigma^{+}\right)\right\}$is a disjoint partition of $\Sigma^{+}$. Hence we conclude that for every $\eta \in \mathcal{B}_{n}\left(\Sigma^{+}\right)$

$$
\mu\left(O\left(\operatorname{Id}, C_{0}(\eta)\right)\right)=\frac{1}{\left|\mathcal{B}_{n}\left(\Sigma^{+}\right)\right|} .
$$


Since every clopen subset of $\Sigma^{+}$is a finite disjoint union of cylinder sets, the above formula determines the normalized invariant mean $\mu$. It is obvious that $\mu$ is a unique normalized invariant mean on $\Gamma^{a}$.

We summarize the above argument:

Theorem 9. Suppose that $\Gamma$ is the groupoid of germs of the adjacency semigroup of a Wieler solenoid and that $\Gamma^{a}$ is its ample semigroup. Then $\Gamma^{a}$ has a unique normalized invariant mean.

The corresponding unique trace on $C^{*}(\Gamma)$ is explained in ([23], Proposition 4.7).

Remark 7. The stable algebras and the stable Ruelle algebras of irreducible Wieler solenoids are studied in [2-4].

Funding: This research was supported by the Basic Science Research Program through the National Research Foundation of Korea (NRF) funded by the Ministry of Education (NRF-2018R1D1A1B07048313).

Conflicts of Interest: The author declares no conflict of interest. The funders had no role in the design of the study; in the collection, analyses, or interpretation of data; in the writing of the manuscript, or in the decision to publish the results.

\section{References}

1. Wieler, S. Smale spaces via inverse limits. Ergod. Theory Dyn. Syst. 2014, 34, 2066-2092. [CrossRef]

2. Brownlowe, N.; Buss, A.; Goncalves, D.; Sims, A.; Whittaker, M. K-Theoretic Duality for Self-Similar Group Actions on Graphs. in preparation.

3. Deely, R.; Goffeng, M.; Mesland, B.; Whittaker, M. Wieler solenoids, Cuntz-Pimsner algebras and K-theory. Ergod. Theory Dyn. Syst. 2018, 38, 2942-2988. [CrossRef]

4. Deeley, R.; Yashinski, A. The stable algebra of a Wieler Solenoid: Inductive limits and K-theory. Ergod. Theory Dyn. Syst. 2019, 1-35. [CrossRef]

5. Bartholdi, L.; Grigorchuk, R.; Nekrashevych, V. From Fractal Groups to Fractal Sets; Fractals in Graz 2001; Grabner, P., Woess, W., Eds.; Birkhäuser: Basel, Switzerland, 2003; pp. 25-118.

6. Grigorchuk, R.; Nekrashevych, V. Self-Similar Groups, Operator Algebras and Schur Complement. J. Mod. Dyn. 2007, 1, 323-370. [CrossRef]

7. Nekrashevych, V. Self-similar inverse semigroups and Smale spaces. Int. J. Algebra Comput. 2006, 16, 849-874. [CrossRef]

8. Exel, R.; Gonçalves, D.; Starling, C. The tiling $C^{*}$-algebra viewed as a tight inverse semigroup algebra. Semigroup Forum. 2012, 84, 229-240. [CrossRef]

9. Kaminker, J.; Putnam, I.; Spielberg, J. Operator Algebras and Hyperbolic Dynamics. In Operator Algebras and Quantum Field Theory (Rome, 1996); Doplicher, S., Longo, R., Roberts, J.E., Zsido, L., Eds.; International Press: Vienna, Austria, 1997; pp. 525-532.

10. Putnam, I. C*-algebras from Smale spaces. Can. J. Math. 1996, 48, 175-195. [CrossRef]

11. Putnam, I. Hyperbolic Systems and Generalized Cuntz-Krieger Algebras; Lecture Notes from Summer School in Operator Algebras, Odense, Denmark; American Mathematical Society: Providence, RI, USA, 1996.

12. Putnam, I.; Spielberg, J. The structure of $C^{*}$-algebras associated with hyperbolic dynamical systems. J. Funct. Anal. 1999, 163, 279-299. [CrossRef]

13. Putnam, I. Lifting factor maps to resolving maps. Israel J. Math. 2005, 146, 253-280. [CrossRef]

14. Exel, R. Partial Dynamical Systems, Fell Bundles and Applications; American Mathematical Society: Providence, RI, USA, 2017; Volume 224.

15. Exel, R.; Pardo, E. The tight groupoid of an inverse semigroup. Semigroup Forum 2016, 92, 274-303. [CrossRef]

16. Exel, R. Inverse semigroups and combinatorial $C^{*}$-algebras. Bull. Braz. Math. Soc. 2008, 39, 191-313. [CrossRef]

17. Lawson, M.V. Inverse Semigroups, the Theory of Partial Symmetries; World Scientific: Singapore, 1998.

18. Lind, D.; Marcus, B. An Introduction to Symbolic Dynamics and Coding; Cambridge University Press: Cambridge, UK, 1995. 
19. Nekrashevych, V. Self-Similar Groups; Math. Surveys and Monographs; American Mathematical Society: Providence, RI, USA, 2005; Volume 117.

20. Nekrashevych, V. C*-algebras and self-similar groups. J. Reine Angew. Math. 2009, 630, 59-123. [CrossRef]

21. Muhly, P.; Renault, J.; Williams, D. Equivalence and isomorphism for groupoid $C^{*}$-algebras. J. Operator Theory 1987, 17, 3-22.

22. Yi, I. Inverse semigroups associated with one-dimensional generalized solenoids. Semigroup Forum 2018, 96, 81-104. [CrossRef]

23. Starling, C. $C^{*}$-algebras of Boolean inverse monoids-traces and invariant means. Doc. Math. 2016, 21, 809-840.

24. Exel, R. Reconstructing a totally disconnected groupoid from its ample semigroup. Proc. Am. Math. Soc. 2010, 138, 2991-3001. [CrossRef]

25. Anantharaman-Delaroche, C.; Renault, J. Amenable Groupoids; Monographies de L'Enseignement Mathématique No. 36; L’Enseignement Mathématique: Geneva, Switzerland, 2000.

(C) 2020 by the author. Licensee MDPI, Basel, Switzerland. This article is an open access article distributed under the terms and conditions of the Creative Commons Attribution (CC BY) license (http:/ / creativecommons.org/licenses/by/4.0/). 